FACTA UNIVERSITATIS (NIŠ)

Ser. Math. Inform. Vol. 35, No 4 (2020), 1231-1237

https://doi.org/10.22190/FUMI2004231M

\title{
GRUNDY DOMINATION SEQUENCES IN GENERALIZED CORONA PRODUCTS OF GRAPHS
}

\author{
Seyedeh Maryam Moosavi Majd and Hamid Reza Maimani**
}

(C) 2020 by University of Niš, Serbia | Creative Commons Licence: CC BY-NC-ND

Abstract. For a graph $G=(V, E)$, a sequence $S=\left(v_{1}, \ldots, v_{k}\right)$ of distinct vertices of $G$ it is called a dominating sequence if $N_{G}\left[v_{i}\right] \backslash \bigcup_{j=1}^{i-1} N\left[v_{j}\right] \neq \varnothing$ and is called a total dom-

inating sequence if $N_{G}\left(v_{i}\right) \backslash \bigcup_{j=1}^{i-1} N\left(v_{j}\right) \neq \varnothing$ for each $2 \leq i \leq k$. The maximum length of (total) dominating sequence is denoted by $\left(\gamma_{g r}^{t}\right) \gamma_{g r}(G)$. In this paper we compute (total) dominating sequence numbers for generalized corona products of graphs.

Keywords: dominating sequence; total dominating sequence; generalized corona products.

\section{Introduction}

In this paper, $G$ is a simple graph with the vertex set $V=V(G)$ and the edge set $E=E(G)$. For notation and graph theoretical terminology, we generally follow [8]. The order $|V|$ and the size $|E|$ of $G$ is denoted by $n=n(G)$ and $m=m(G)$, respectively. For every vertex $v \in V$, the open neighborhood $N_{G}(v)$ of $v$ is the set $\{u \in V(G): u v \in E(G)\}$ and the closed neighborhood of $v$ is the set $N_{G}[v]=N_{G}(v) \cup\{v\}$. The degree of a vertex $v \in V$ is $\operatorname{deg}_{G}(v)=d_{G}(v)=\left|N_{G}(v)\right|$. The minimum degree and the maximum degree of a graph $G$ are denoted by $\delta=\delta(G)$ and $\Delta=\Delta(G)$, respectively. We write $P_{n}$ for the path of order $n, C_{n}$ for the cycle of order $n$, and $K_{n}$ for the complete graph of order $n$. A subset $D$ of $V(G)$ is called a dominating set of $G$ if every vertex of $G$ is either in $D$ or adjacent to at least one vertex in $D$. The domination number of $G$, denoted by $\gamma(G)$, is the number of vertices in a smallest dominating set of $G$. A total dominating set of $G$ is a set $D$ of vertices of $G$ such that every vertex is adjacent to a vertex in $D$. The total domination number of $G$, denoted by $\gamma_{t}(G)$, is the minimum cardinality of a total dominating set. A dominating set of cardinality $\gamma(G)\left(\gamma_{t}(G)\right)$ is called a $\gamma$-set

Received July 12, 2020; accepted August 21, 2020

2020 Mathematics Subject Classification. Primary 05C69; Secondary 05C76

* Corresponding Author 
$\left(\gamma_{t^{-}}\right.$set $)$. For further information about various domination sets in graphs, we refer reader to $[9,10]$.

Let $G$ be a graph of order $n$ and let $H_{1}, H_{2}, \ldots, H_{n}$, be $n$ graphs. The generalized corona product, is the graph obtained by taking one copy of graphs $G, H_{1}, H_{2}, \cdots, H_{n}$ and joining the $i$ th vertex of $G$ to every vertex of $H_{i}$. This product is denoted by $G \circ \wedge_{i=1}^{n} H_{i}$. If each $H_{i}$ is isomorphic to a graph $H$, then generalized corona product is called the corona product of $G$ and $H$ and is denoted by $G \circ H$.

Let $G$ be a graph of size $m$ and $H$ be a graph. The edge corona product, denoted by $G \diamond H$, is the graph obtained by taking one copy of $G$ and $m$ copies of $H$, and then joining two end-vertices of the $i$ th edge $e_{i}$ of $G$ to every vertex of $i$ th copy of $H$. The neighborhood corona, denoted by $G \star H$, is the graph obtained by taking $n$ copies of $H$ and for each $i, 1 \leq i \leq n$, the $i$ th copy of $H$ being adjacent to vertices of $N_{G}\left[v_{i}\right]$. It is not difficult to see that $G \diamond H$ is the same as $G \circ \wedge_{i=1}^{n} H_{i}$, where each $H_{i}$ is a disjoint union of $\operatorname{deg}\left(v_{i}\right)$ copies of $H$ and $G \star H$ is the same as $G \circ \wedge_{i=1}^{n} H_{i}$, where each $H_{i}$ is a disjoint union of $\operatorname{deg}\left(v_{i}\right)+1$ copies of $H$.

Based on the domination number and the total domination number, various Grundy domination invariants have been introduced in recent years by some authors $[1,5,6]$ and then they continued the study of these concepts in $[3,2,4,7]$.

In [5] the first type of Grundy dominating sequence was introduced. Let $S=$ $\left(v_{1}, \ldots, v_{k}\right)$ be a sequence of distinct vertices of a graph $G$. The corresponding set $\left\{v_{1}, \ldots, v_{k}\right\}$ of vertices from the sequence $S$ will be denoted by $\widehat{S}$. A sequence $S=\left(v_{1}, \ldots, v_{k}\right)$ is called a closed neighborhood sequence if, for each $i$,

$$
N_{G}\left[v_{i}\right] \backslash \bigcup_{j=1}^{i-1} N_{G}\left[v_{j}\right] \neq \varnothing .
$$

If for a closed neighborhood sequence $S$, the set $\widehat{S}$ is a dominating set of $G$, then $S$ is called a dominating sequence of $G$. Clearly, if $S=\left(v_{1}, v_{2}, \ldots, v_{k}\right)$ is a dominating sequence for $G$, then $k \geq \gamma(G)$. We call the maximum length of a dominating sequence in $G$ the Grundy domination number of $G$ and denote it by $\gamma_{g r}(G)$. The corresponding sequence is called a Grundy dominating sequence of $G$ or $\gamma_{g r}$-sequence of $G$.

Total dominating sequences were introduced in [6], when $G$ is a graph without isolated vertices. Using the same notation as in the previous paragraph, we say that a sequence $S=\left(v_{1}, \ldots, v_{k}\right)$ is called an open neighborhood sequence if, for each $2 \leq i \leq k$,

$$
N_{G}\left(v_{i}\right) \backslash \bigcup_{j=1}^{i-1} N_{G}\left(v_{j}\right) \neq \varnothing .
$$

Any open neighborhood sequence $S$, where $\widehat{S}$ is a total dominating set is called a total dominating sequence. The maximum length of a total dominating sequence in $G$ is called the Grundy total domination number of $G$ and denoted by $\gamma_{g r}^{t}(G)$. 
The corresponding sequence is called a Grundy total dominating sequence of $G$ or a $\gamma_{g r}^{t}$-total sequence.

An additional variant of the Grundy (total) domination number was introduced in [1]. Let $G$ be a graph without isolated vertices. A sequence $S=\left(v_{1}, \ldots, v_{k}\right)$, where $v_{i} \in V(G)$, is called a $Z$ - sequence if for each $i$,

$$
N_{G}\left(v_{i}\right) \backslash \bigcup_{j=1}^{i-1} N_{G}\left[v_{j}\right] \neq \varnothing
$$

Then the $Z$-Grundy domination number $\gamma_{g r}^{Z}(G)$ of the graph $G$ is the length of a longest $Z$-sequence.

Let $S_{1}=\left(v_{1}, \ldots, v_{n}\right)$ and $S_{2}=\left(u_{1}, \ldots, u_{m}\right), n, m \geq 1$, be two sequences in $G$, with $\widehat{S_{1}} \cap \widehat{S_{2}}=\varnothing$. The concatenation of $S_{1}$ and $S_{2}$ is defined as the sequence $S_{1} \oplus S_{2}=\left(v_{1}, \ldots, v_{n}, u_{1}, \ldots, u_{m}\right)$. Clearly $\oplus$ is an associative operation on the set of all sequences, but is not commutative. If $S_{2}=\{v\}$, then $S_{1} \oplus S_{2}$ is denoted by $S_{1} \oplus v$.

In the next section, we compute Grundy domination numbers for generalized corona products of graphs and based on, we find Grundy domination numbers of edge and neighborhood corona products of graphs.

\section{Main Results}

In this section we give the exact value of (total) Grundy domination numbers for generalized corona products, and compute them for corona product of some special graphs. First we state two necessary known propositions.

Proposition 2.1. [6] For $n \geq 4$ even, $\gamma_{g r}^{t}\left(P_{n}\right)=n$ and $\gamma_{g r}^{t}\left(C_{n}\right)=n-2$, while for $n \geq 3$ odd, $\gamma_{g r}^{t}\left(P_{n}\right)=\gamma_{g r}^{t}\left(C_{n}\right)=n-1$.

Proposition 2.2. $\quad[5,1]$ For $n \geq 3, \gamma_{g r}\left(C_{n}\right)=\gamma_{g r}^{Z}\left(C_{n}\right)=n-2$, while for $n \geq 2$, $\gamma_{g r}\left(P_{n}\right)=\gamma_{g r}^{Z}\left(P_{n}\right)=n-1$.

we are now state and proof the our first main result.

Theorem 2.1. Let $G$ and $H_{1}, H_{2}, \ldots, H_{n}$ be $n+1$ graphs without isolated vertices. Then

$$
\gamma_{g r}\left(G \circ \wedge_{i=1}^{n} H_{i}\right)=\sum_{i=1}^{n} \gamma_{g r}\left(H_{i}\right)+\gamma_{g r}^{Z}(G) .
$$

Proof. Set $K=G \circ \wedge_{i=1}^{n} H_{i}$. Let $S=\left(v_{1}, \ldots, v_{k}\right)$ be a $Z$-Grundy sequence of $G$ and $S_{i}$ be a $\gamma_{g r}$-sequence of $H_{i}$ for $1 \leq i \leq n$. It is not difficult to see that

$$
S_{1} \oplus v_{1} \oplus S_{2} \oplus v_{2} \oplus \ldots \oplus S_{k} \oplus v_{k} \oplus S_{k+1} \oplus S_{k+2} \oplus \ldots \oplus S_{n}
$$


is a dominating sequence for $K$. This implies that $\gamma_{g r}(K) \geq \sum_{i=1}^{n} \gamma_{g r}\left(H_{i}\right)+\gamma_{g r}^{Z}(G)$.

Let $T$ be a $\gamma_{g r}$-sequence of $K$ such that $|\widehat{T} \cap V(G)|$ is minimum among all $\gamma_{g r}$-sequences. Suppose that $\widehat{T} \bigcap V(G)=\left\{v_{1}, \ldots, v_{t}\right\}$, where $\left(v_{1}, \ldots, v_{t}\right)$ is a subsequence of $T$. If $t>\gamma_{g r}^{Z}(G)$, then $\left(v_{1}, \ldots, v_{t}\right)$ is not a $Z$-sequence for $G$ and thus, there exists $1 \leq l \leq t$ such that $N_{G}\left(v_{l}\right) \backslash \bigcup_{i=1}^{l-1} N_{G}\left[v_{i}\right]=\varnothing$. But $N_{K}\left[v_{l}\right] \backslash \bigcup_{i=1}^{l-1} N_{K}\left[v_{i}\right] \neq \varnothing$, since $\left(v_{1}, \ldots, v_{t}\right)$ is a sub-sequence of $T$. If $\widehat{T} \bigcap V\left(H_{l}\right) \neq \varnothing$, then there exists an element $z \in V\left(H_{l}\right)$ such that one of the $\left(v_{1}, \ldots, v_{l}, z\right)$ or $\left(v_{1}, \ldots, v_{i-1}, z, v_{i}, \ldots, v_{l}\right)$ is a subsequence of $T$. If $\left(v_{1}, \ldots, v_{l}, z\right)$ is a subsequence of $T$, then $N_{K}[z] \backslash \bigcup_{i=1}^{l} N_{K}\left[v_{i}\right]=$ $\varnothing$, which is a contradiction. Hence $\left(v_{1}, \ldots, v_{i-1}, z, v_{i}, \ldots, v_{l}\right)$ is a subsequence of $T$. Therefore there exists $x \in N_{K}\left[v_{l}\right] \backslash \bigcup_{i=1}^{l-1} N_{K}\left[v_{i}\right] \bigcup N_{K}[z]$. Since $v_{l} \in N_{K}[z]$ and $N_{G}\left(v_{l}\right) \backslash \bigcup_{i=1}^{l-1} N_{G}\left[v_{i}\right]=\varnothing$, we conclude that $x \neq v_{l}$. In addition, $x \in V\left(H_{l}\right)$ and $x, z$ are not adjacent vertices, and $x \notin \widehat{T}$. Now, by replacing $v_{l}$ by $x$ in $T$, we obtain a $\gamma_{g r}$-sequence $T^{\prime}$, such that $\left|\widehat{T^{\prime}} \cap V(G)\right|<|\widehat{T} \cap V(G)|$, which is a contradiction. Hence $\widehat{T} \cap V\left(H_{l}\right)=\varnothing$. Again consider a vertex $x \in V\left(H_{l}\right)$ and put $x$ instead of $v_{l}$ in $T$. Then we obtain a $\gamma_{g r}$-sequence $T^{\prime}$ such that the size of intersection of $\widehat{T^{\prime}}$ and $V(G)$ is less than the size of intersection of $\widehat{T}$ and $V(G)$. This is a contradiction and so we conclude that $|\hat{T} \bigcap V(G)| \leq \gamma_{g r}^{Z}(G)$. It is not difficult to see $\left|\widehat{T} \cap V\left(H_{i}\right)\right| \leq \gamma_{g r}\left(H_{i}\right)$ for $1 \leq i \leq n$ and thus $\gamma_{g r}(K) \leq \sum_{i=1}^{n} \gamma_{g r}\left(H_{i}\right)+\gamma_{g r}^{Z}(G)$.

The following corollary is an easy consequence of Theorem 2.1 and Proposition 2.2 .

Corollary 2.1. For $n, m \geq 3$

$$
\begin{aligned}
& \gamma_{g r}\left(C_{n} \circ C_{m}\right)=n(m-1)-2, \gamma_{g r}\left(P_{n} \circ P_{m}\right)=m n-1, \\
& \gamma_{g r}\left(C_{n} \circ P_{m}\right)=n m-2, \gamma_{g r}\left(P_{n} \circ C_{m}\right)=n(m-1)-1 .
\end{aligned}
$$

we are now stat and proof our second main result.

Theorem 2.2. Let $G$ and $H_{1}, H_{2}, \ldots, H_{n}$ be graphs without isolated vertices. Then

$$
\gamma_{g r}^{t}\left(G \circ \wedge_{i=1}^{n} H_{i}\right)=\sum_{i=1}^{n} \gamma_{g r}^{t}\left(H_{i}\right)+\gamma_{g r}^{Z}(G) .
$$

Proof. Consider the sequence

$$
T=S_{1} \oplus v_{1} \oplus S_{2} \oplus v_{2} \oplus \ldots \oplus S_{k} \oplus v_{k} \oplus S_{k+1} \oplus S_{k+2} \oplus \ldots \oplus S_{n},
$$

where $S=\left(v_{1}, \ldots, v_{k}\right)$ is a $Z$-Grundy sequence of $G$ and $S_{i}$ 's are $\gamma_{g r}^{t}$-sequences of $H_{i}$ 's for $1 \leq i \leq n$. We show that $T$ is a $\gamma_{g r}^{t}$-sequence for $K=G \circ \wedge_{i=1}^{n} H_{i}$. Let $x \in \widehat{T}$. Hence there exists either $1 \leq i \leq n$ such that $x \in \widehat{S}_{i}$ or $1 \leq j \leq k$ for which $x=v_{j}$. If $x=v_{j}$, then there exists $y \in N_{G}\left(v_{j}\right) \backslash \bigcup_{t=1}^{j-1} N_{G}\left[v_{t}\right]$. Hence $y \neq v_{t}$ for 
$1 \leq t \leq j-1$ and therefore $y \in N_{K}\left(v_{j}\right) \backslash \bigcup_{t=1}^{j-1} N_{K}\left[v_{t}\right] \bigcup\left(\bigcup_{t=1}^{j} N_{k}\left[S_{t}\right]\right)$. This implies that

$$
N_{K}\left(v_{j}\right) \backslash \bigcup_{t=1}^{j-1} N_{K}\left[v_{t}\right] \bigcup\left(\bigcup_{t=1}^{j} N_{K}\left[S_{t}\right]\right) \neq \varnothing
$$

The same argument can be apply when $x \in \widehat{S}_{i}$. Since clearly $\widehat{T}$ is a total dominating set, we conclude that $T$ is a total dominating sequence of $G$. Hence

$$
\gamma_{g r}^{t}(K) \geq \sum_{i=1}^{n} \gamma_{g r}^{t}\left(H_{i}\right)+\gamma_{g r}^{Z}(G)
$$

Now suppose that $T$ is a $\gamma_{g r}^{t}$-sequence of $K$ such that $|\widehat{T} \bigcap V(G)|$ is minimum among all $\gamma_{g r}^{t}$-sequences of $G$. Suppose that $\widehat{T} \bigcap V(G)=\left\{v_{1}, \ldots, v_{t}\right\}$ and $t>$ $\gamma_{g r}^{Z}(G)$. Hence $\left(v_{1}, \ldots, v_{t}\right)$ is not a $Z$-sequence for $G$. Therefore, there exists $1 \leq$ $l \leq t$ such that $N_{G}\left(v_{l}\right) \backslash \bigcup_{i=1}^{l-1} N_{G}\left[v_{i}\right]=\varnothing$. If $\widehat{T} \bigcap V\left(H_{l}\right)=\varnothing$, then by replacing $v_{l}$ by $x \in V\left(H_{l}\right)$, we can construct a $\gamma_{g r}^{t}$-sequence $T^{\prime}$ such that $\left|\widehat{T^{\prime}} \cap V(G)\right|<|\widehat{T} \cap V(G)|$, which is a contradiction. Hence $\widehat{T} \cap V\left(H_{l}\right) \neq \varnothing$. If there exists $x \in \widehat{T} \cap V\left(H_{l}\right)$ such that $x$ appears after $v_{l}$ in the sequence $T$, then $\left(v_{l}, x\right)$ is a subsequence of $T$ and $N_{K}(x) \backslash N_{K}\left(v_{l}\right) \neq \varnothing$. Since $N_{G}\left(v_{l}\right) \backslash \bigcup_{i=1}^{l-1} N_{G}\left[v_{i}\right]=\varnothing$, we conclude that $N_{K}(x) \backslash N_{K}\left(v_{l}\right)=\left\{v_{l}\right\}$ and hence $\widehat{T} \cap V\left(H_{l}\right)=\{x\}$. Now choose $y \in N(x)$ and replace $v_{l}$ by $y$ in $T$. Again we obtain a $\gamma_{g r}^{t}$-sequence $T^{\prime}$ such that $\left|\widehat{T^{\prime}} \cap V(G)\right|<$ $|\widehat{T} \cap V(G)|$, which is a contradiction. Hence all elements of $\widehat{T} \cap V\left(H_{l}\right)$ appear before $v_{l}$ in the sequence $T$. Hence there exists $y \in V\left(H_{l}\right)$ such that $y \in N_{K}\left(v_{l}\right) \backslash$ $\bigcup_{x \in \widehat{T} \cap V\left(H_{l}\right)} N_{K}(x)$. Since $\operatorname{deg}_{H_{l}}(y) \geq 1$, there exists $z \in V\left(H_{l}\right)$ which is adjacent to $y$. Clearly $z \notin \widehat{T}$ and by changing $v_{l}$ with $z$, we get a $\gamma_{g r}^{t}$-sequence $T^{\prime}$ such that $\left|\widehat{T^{\prime}} \cap V(G)\right|<|\widehat{T} \cap V(G)|$, which is a contradiction. This argument implies that $|\widehat{T} \bigcap V(G)| \leq \gamma_{g r}^{Z}(G)$. One can easily check that $\left|\widehat{T} \bigcap V\left(H_{i}\right)\right| \leq \gamma_{g r}^{t}\left(H_{i}\right)$ for $1 \leq i \leq n$ and so we conclude that $\gamma_{g r}^{t}(K) \leq \sum_{i=1}^{n} \gamma_{g r}^{t}\left(H_{i}\right)+\gamma_{g r}^{Z}(G)$.

Corollary 2.2. Let $G$ be a graph of order $n$ and size $m$ and $H$ be a graph without isolated vertices. Then $\gamma_{g r}(G \diamond H)=2 m \gamma_{g r}(H)+\gamma_{g r}^{Z}(G)$ and $\gamma_{g r}^{t}(G \star H)=(2 m+$ $n) \gamma_{g r}^{t}(H)+\gamma_{g r}^{Z}(G)$.

Proof. Note that $G \diamond H$ is the same as $G \circ \wedge_{i=1}^{n} H_{i}$, where $H_{i}$ is the disjoint union of $\operatorname{deg}\left(v_{i}\right)$ copies of $H$. Hence by Theorem 2.1,

$$
\gamma_{g r}(G \diamond H)=\gamma_{g r}\left(G \circ \wedge_{i=1}^{n} H_{i}\right)=\sum_{i=1}^{n} \gamma_{g r}\left(H_{i}\right)+\gamma_{g r}^{Z}(G)=2 m \gamma_{g r}(H)+\gamma_{g r}^{Z}(G) .
$$

The proof of the second part of the corollary is similar.

Corollary 2.3. Let $G$ be a connected graph of order $n$. Then $\gamma_{g r}^{t}\left(G \circ K_{1}\right)=2 n$. 
Proof. Suppose that $V(G)=\left\{v_{1}, \ldots, v_{n}\right\}$ is the vertex set of $G$. It is not difficult to see that sequence $\left(u_{1}, u_{2}, \ldots, u_{n}, v_{1}, v_{2}, \ldots, v_{n}\right)$, where $u_{i}$ is the vertex of $K_{1}$, which is adjacent to $v_{i}$, is a Grundy total domination sequence of $G \circ K_{1}$.

Corollary 2.4. Let $G$ be a nontrivial connected graph of order $n$. Then $\gamma_{g r}^{t}(G \circ$ $H)=n \gamma_{g r}^{t}(H)+\gamma_{g r}^{Z}(G)$, for any nontrivial connected graph $H$.

As a similar argument to proof of Theorem 2.1, we can find the $Z$-Grundy domination number of corona product of graphs.

Theorem 2.3. Let $G$ and $H_{1}, H_{2}, \ldots, H_{n}$ be $n+1$ graphs without isolated vertices. Then

$$
\gamma_{g r}^{Z}\left(G \circ \wedge_{i=1}^{n} H_{i}\right)=\sum_{i=1}^{n} \gamma_{g r}^{Z}\left(H_{i}\right)+\gamma_{g r}^{Z}(G)
$$

\section{REF E R E N C E S}

1. B. Brešar, Cs. Bujtas, T. Gologranc, S. Klavzar, G. Kosmrlj, B. Patkos, Z. Tuza and M. Vizer, Grundy dominating sequences and zero forcing sets, Discrete Optim. 26 (2017), 66-77.

2. B. Brešar, C. Bujtas, T. Gologranc, S. Klavzar, G. Kosmrlj, B. Patkos, Z. Tuza, M. Vizer, Dominating sequences in grid-like and toroidal graphs Electron. J. Combin., 23 (2016), P4.34 (19 pages).

3. B. Brešar, T. Gologranc and T. Kos, Dominating sequences under atomic changes with applications in Sierpinski and interval graphs, Appl. Anal. Discrete Math. 10 (2016), 518-531.

4. B. Brešar, Kos and Terros, Grundy domination and zero forcing in Kneser graphs, Ars Math. Contemp., 17(2019), 419-430.

5. B. Brešar, T. Gologranc, M. Milanič, D. F. Rall, R. Rizzi, Dominating sequences in graphs. Discrete Math. 336 (2014), 22-36.

6. B. Brešar, M. A. Henning, D. F. Rall, Total dominating sequences in graphs. Discrete Math. 339 (2016) 1165-1676.

7. B. Brešar, T. Kos, G. Nasini, P. Torres, Total dominating sequences in trees, split graphs, and under modular decomposition, Discrete Optim., 28(2018), 16-30.

8. G. Chartrand, L. Lesniak, Graphs and digraphs, Third Edition, CRC Press,(1996).

9. T. W. Haynes, S. Hedetniemi, P. Slater, Fundamentals of Domination in Graphs, CRC Press, (1998).

10. M. A. Henning and A. Yeo, Total domination in graphs, (Springer Monographs in Mathematics.) ISBN-13: 987-1461465249 (2013).

Seyedeh Maryam Moosavi Majd

Department of Mathematics

Science and Research Branch, Islamic Azad University

Tehran, Iran

moosavi.majd@gmail.com 
Hamid Reza Maimani

Mathematics Section, Department of Basic Sciences

Shahid Rajaee Teacher Training University

P.O. Box 16785-163

Tehran, Iran

maimani@ipm.ir 